\title{
Influência do Estresse Psicossocial no Lúpus Eritematoso Sistêmico $\left.{ }^{*}\right)$
}

\section{Influence of the Psychosocial Stress on Systemic Lupus Erythematosus}

\author{
Fabiano Gonçalves Nery ${ }^{(1)}$, Eduardo Ferreira Borba ${ }^{(2)}$ e Francisco Lotufo Neto ${ }^{(3)}$
}

\section{RESUMO}

Vários estudos mostram que doenças clínicas podem piorar sob a influência de fatores psicologicamente estressantes. Entretanto, poucos estudos foram feitos para estabelecer esta correlação no lúpus eritematoso sistêmico (LES). No presente artigo, revisamos estudos sobre a associação ou influência de estresse psicológico ou social em pacientes com LES. Os estudos foram identificados por uma busca no Medline, através dos termos "stress", "lupus", "disease activity" e "flare". Foram selecionados 14 artigos que apresentaram uma grande variação na metodologia empregada. A maioria falhou em encontrar associações entre a presença de estresse e a piora da atividade laboratorial ou clínica do LES. Apesar disso, foi identificada uma associação positiva entre presença de estresse e pior percepção da saúde física pelo paciente, através de medidas subjetivas. Três estudos de modelo laboratorial de estresse psicológico mostraram que a resposta biológica ao estresse em pacientes com LES difere de controles normais e pacientes com artrite reumatóide. Apenas um estudo mostrou que estresse diário em relações ou obrigações sociais no mês prévio precedia a exacerbação do LES.

Palavras-chave: estresse, estresse psicológico, lúpus eritematoso sistêmico, atividade da doença.

\section{INTRODUÇÃO}

A influência do estresse no curso de doenças orgânicas, embora desperte interesse e seja de grande importância no acompanhamento destas, é tema pouco explorado na literatura,

\begin{abstract}
Several studies demonstrate that clinical diseases may worsen under the influence of psychologically stressing factors. However few studies have been realized to establish this correlation in systemic lupus erythematosus (SLE). In the present article, studies about the association and influence of psychological or social stress in patients with SLE were reviewed. Studies were identified by a Medline search, relating the terms "stress", "lupus", "disease activity", and "flare". Fourteen articles were selected but presented a great variability in the employed methodology. The majority failed to find associations between the presence of stress and worsening of clinical or laboratorial activity of SLE. Nevertheless, association between presence of stress and worse perception of physical health by the patient, through subjective measures of health was identified. Three studies of laboratorial model of psychological stress showed that biological response to stress in SLE patients differs from normal controls and patients with rheumatoid arthritis. Only one study showed that daily stress in social relationships or social duties in the previous month preceded SLE exacerbation.
\end{abstract}

Keywords: stress, psychological stress, systemic lupus erythematosus, disease activity.

principalmente no que se refere às doenças reumáticas. Entretanto, nota-se cada vez mais a importância de sua participação pois há evidências de que fatores psicossociais estressantes interferem diretamente numa série de situações patológicas como processos de cicatrização ou no surgimento

\footnotetext{
* Departamento e Instituto de Psiquiatria da Faculdade de Medicina da Universidade de São Paulo (FMUSP) e Disciplina de Reumatologia do Hospital das Clínicas da FMUSP, São Paulo-SP, Brasil. Este trabalho recebeu bolsa de apoio à pesquisa da Fundação de Amparo à Pesquisa do Estado de São Paulo (FAPESP). Recebido em 26/6/2004. Aprovado, após revisão, em 12/7/2004.

1. Pós-graduando em Psiquiatria pela FMUSP, São Paulo-SP, Brasil.

2. Médico assistente do Serviço de Reumatologia do HC/FMUSP, São Paulo-SP, Brasil.

3. Professor associado do Departamento de Psiquiatria da FMUSP, São Paulo-SP, Brasil.

Endereço para correspondência: Fabiano Gonçalves Nery. Secretaria de Pós-Graduação do Programa de Psiquiatria - Departamento de Psiquiatria da Faculdade de Medicina da Universidade de São Paulo (FMUSP). Rua Dr. Ovídio Pires de Campos, 785, São Paulo-SP, CEP 05430-010. E-mail: fgnery@uol.com.br
} 
e exacerbação de doenças cardiovasculares, câncer, infecções agudas, síndrome da imunodeficiência adquirida (AIDS), asma e até mesmo na artrite reumatóide ${ }^{(1-12)}$.

Neste sentido, alguns estudos demonstraram, através de diferentes métodos, que o estresse ambiental promove alterações neuroendócrinas e imunológicas, como o aumento de cortisol e de catecolaminas, além de significativa redução no número e na atividade de linfócitos ${ }^{(13)}$. Estes dados geraram grande interesse diante da possibilidade de que o estresse possa apresentar repercussão em doenças clínicas, principalmente naquelas de base imunológica, como o lúpus eritematoso sistêmico (LES).

\section{O SISTEMA DE RESPOSTA AO ESTRESSE}

Cannon foi o primeiro a observar que em situações de frio intenso, falta de oxigênio e fortes emoções, o sistema nervoso simpático libera substâncias que determinam reações orgânicas adaptativas, denominadas a partir de então de "luta ou fuga" (fight or flight) ${ }^{(14)}$. Posteriormente, em 1936, Hans Selye descreveu a síndrome de adaptação geral, que envolve um conjunto de reações do organismo a agressões de ordem física, psíquica, infecciosa ou ambiental, capazes de perturbar a sua homeostase ${ }^{(15)}$. Mais tarde, em 1964, George F. Solomon publicou um artigo de suma importância, expondo uma teoria que integra os conhecimentos sobre a influência das emoções na imunidade e na doença ${ }^{(16)}$. Somente em 1975 surgiu o conceito da psiconeuroimunologia, introduzido por Ader a partir dos achados de imunossupressão de ratos com sacarina, após o seu condicionamento com estímulos aversivos, e sobre o aumento da sobrevida de ratos com lúpus eritematoso sistêmico, tratados apenas com sacarina, após serem condicionados com estímulos aversivos ${ }^{(17)}$. Progressivamente, acumularam-se trabalhos nesta área, sendo cada vez mais reconhecida a influência do ambiente, das emoções e do comportamento na saúde fisica.

A idéia central é a de que eventos estressantes, físicos ou psicológicos desencadeiem uma resposta orgânica comum, levando a prejuízos ou lesões observados no organismo estressado. Atualmente existem fortes evidências que apontam as alterações no eixo hipotálamo-hipófise-adrenal (eixo HHA) e no sistema nervoso simpático (SNS) como importante marcadores de resposta ao estresse e mediadores de mudanças fisiopatólogicas ${ }^{(18)}$. Desta forma, regiões cerebrais como a amígdala e o córtex pré-frontal, envolvidas nas funções de percepção e processamento do medo, ansiedade, memória emocional, comportamento executivo complexo e flexibilidade afetiva, interagem bidirecionalmente com outras regiões ligadas à mediação de respostas endócrinas e simpáticas, como o hipotálamo e o locus ceruleus $^{(19,20)}$. Como conseqüência do evento estressor, existe um aumento da secreção de noradrenalina pelo locus ceruleus, e do hormônio liberador de corticotropina $(\mathrm{CRH})$ pelo hipotálamo. O CRH age na hipófise que libera o hormônio adrenocorticotrófico (ACTH), que induz as glândulas adrenais a produzirem cortisol. Sabe-se que o aumento de cortisol e de noradrenalina circulantes é essencial para a resposta de estresse, e seus efeitos agudos são o aumento da produção e utilização de energia, da freqüência cardíaca e pressão arterial, além da inibição de funções neurovegetativas, como crescimento, reprodução e imunidade. Em última análise, essas alterações preparam o organismo para o confronto biológico de situações de alarme (estressantes) ${ }^{(21)}$.

Embora este tipo de resposta seja extremamente útil, caso se torne persistente e crônico pode levar a consequiências deletérias para o organismo, pois promove resistência periférica à insulina, deposição de gordura, osteopenia e osteoporose, além de diminuição da função imunitária de células $T$ helper ${ }^{(21)}$.

Sabe-se que os órgãos e estruturas que participam do sistema imunológico são inervados pelo SNS, e que suas células possuem receptores de glicocorticóides, explicando a alteração de suas funções na resposta ao estresse. Glicocorticóides e catecolaminas influenciam reconhecidamente múltiplos aspectos da resposta imune celular e humoral, bem como a produção e liberação de citocinas ${ }^{(22)}$.

Desta forma, os diversos estudos laboratoriais de estresse agudo mostraram evidências que ligam fatores psicológicos e alterações endócrinas a repercussões imunológicas. Estressores laboratoriais agudos produzem alterações imunes transitórias, decorrentes da liberação aguda de catecolaminas na circulação, promovendo muito mais uma redistribuição de linfócitos circulantes no sangue periférico (um processo chamado trafficking) do que alterando seu número total, mas que retornam ao normal uma hora após cessar o estressor ${ }^{(23)}$. Outros estudos também demostraram que a função imune piora sob estressores naturais agudos e de curto prazo, como exames escolares. $\mathrm{Na}$ vigência destes, relata-se aumento do tempo de cicatrização de feridas, maior susceptibilidade a infecções virais agudas, e menor soroconversão à vacinas ${ }^{(1,6,7,24)}$.

Por outro lado, os efeitos do estresse crônico são menos conhecidos, mas estes também podem ter efeitos deletérios sobre a função imune. Situações como cuidar de um parente 
com demência de Alzheimer, conflito conjugal, desemprego ou desastres naturais estiveram associados com diversas alterações da função imune, como diminuição de soroconversão à vacinas, menor resposta blastogênica a mitógenos, proliferação de linfócitos diminuída, menor reação tuberculínica e redução de subpopulações de linfócitos (como CD4 e CD8) ${ }^{(25-29)}$. Sabe-se ainda que tanto estressores agudos como crônicos têm sido associados ao aumento da produção e liberação de citocinas pró-inflamatórias, como interleucinas 1 e $6^{(30)}$.

Além disso, também é sugerido que alterações comportamentais freqüentemente identificadas no indivíduo estressado, como diminuição ou aumento de atividade física, de horas de sono, mudanças na dieta e no uso de álcool, cafeína, nicotina e drogas, podem por si só ter efeitos deletérios à função imune ${ }^{(31)}$.

\section{INFLUÊNCIA DO ESTRESSE NO LES}

É interessante notar que tanto reumatologistas experientes quanto os próprios pacientes freqüentemente relacionam a piora ou surgimento do LES com alguma situação de "estresse". Por outro lado, é bem definido atualmente que a doença possui evolução tipicamente marcada por períodos variáveis de remissão e exacerbação, e que sofre influência de alguns fatores como exposição solar, hormônios, drogas, e agentes infecciosos ${ }^{(32)}$.

Porém, até 1991, todos os estudos que tiveram o intuito de verificar a relação do estresse como deflagrador ou intensificador do quadro clínico do LES foram retrospectivos e baseados na constatação subjetiva dos próprios pacientes com LES, que atribuíam a eventos estressantes ou fatores psicológicos a causa de sua doença ${ }^{(33-35)}$. De fato, através destas formas de abordagem, não é de se espantar que os próprios pacientes atribuam a eventos externos a piora de sua sintomatologia ou mesmo do aparecimento do quadro clínico. Desta forma, estudos de metodologia apropriada se tornam necessários para verificar essas associações de forma mais adequada.

É importante salientar que apenas três trabalhos demostraram alterações na resposta imune em pacientes com LES, após exposição a um estressor psicológico agudo, em condições laboratoriais ${ }^{(36-38)}$. O primeiro estudo com este objetivo foi realizado em 1992 por Hinrichsen et al., que verificaram a influência de um teste neuropsicológico de estresse no LES, através das alterações de catecolaminas plasmáticas, cortisol e na distribuição de subpopulações de linfócitos em 14 pacientes com LES comparados a 10 pacientes sem colagenose mas tratados com prednisona e 14 controles saudáveis. O estresse neuropsicológico induziu um aumento significativo nos níveis plasmáticos de adrenalina e noradrenalina, quando comparados aos parâmetros basais nos três grupos estudados, porém os níveis de cortisol permaneceram inalterados. $\mathrm{O}$ aumento das catecolaminas estava significativamente associado com a mobilização celular nos controles e nos indivíduos tratados com prednisona, mas este não foi observado nos pacientes com LES. Da mesma forma, as células CD19+ aumentaram significativamente em número com relação aos valores basais nos controles e nos tratados com prednisona, e permaneciam inalterados nos pacientes com LES. Os autores concluem que pacientes com lúpus demonstram redução da mobilização celular em razão do estresse psicológico, apesar de apresentarem alterações hormonais semelhantes aos outros dois grupos estudados ${ }^{(36)}$.

Posteriormente, Pawlak et al. também procuraram identificar alterações na freqüência cardíaca, pressão arterial, concentração de catecolaminas, subpopulações de linfócitos, atividade de células natural killer (NK) e expressão de $\beta$-adrenoreceptores nos monócitos de sangue periférico após exposição a uma situação de estresse, no caso, falar em público. Estudando 15 pacientes com LES comparados a 15 controles normais, observaram um aumento do número de células NK e do número de $\beta$-adrenoreceptores nos monócitos de sangue periférico induzidos por estresse nestes dois grupos, porém foi significativamente menor nos pacientes com LES, e não existiram diferenças nas respostas psicológicas, cardiovasculares e neuroendócrinas. Os autores concluem que pacientes com LES diferem de controles saudáveis nas respostas imunes induzidas por estresse $^{(37)}$.

Finalmente, este mesmo grupo também investigou alterações na resposta imune (concentração de catecolaminas, número de leucócitos, subpopulações de linfócitos, produção de citocinas e de células NK) após exposição ao estresse, desta vez comparando pacientes com LES $(n=7)$, controles saudáveis ( $n=15)$, e pacientes com outra patologia reumatológica, a artrite reumatóide $(\mathrm{n}=9)$. Interessantemente, os pacientes com LES demonstraram aumento significativamente menor no número de leucócitos circulantes e de células NK. Além disso, também puderam observar aumento significativo no número de células produtoras de interleucina 4 , bem como diminuição na produção de interleucina 10 , respectivamente ligadas à indução de linfócitos B auto-reativos e à imunossupressão. Esses achados fizeram os autores postularem que talvez 
exista uma proliferação e expansão de linfócitos B autoreativos, e conseqüentemente um aumento dos níveis de autoanticorpos, na tentativa de explicar uma possível exacerbação do LES sob situações de estresse agudo ${ }^{(38)}$.

Os demais trabalhos sobre este tema objetivaram verificar uma provável associação do estado de atividade do LES com o estresse ambiental na forma de eventos de vida (ocasionais e mais intensos) e de estressores cotidianos (corriqueiros e mais leves).

Nesta linha de investigação, merece destaque o relato de Schubert et al. por apresentar uma metodologia peculiar. Estes autores acompanharam uma única paciente com LES durante pouco mais de dois meses, verificando estressores cotidianos semanalmente e realizando diariamente medidas de neopterina urinária, um parâmetro imunológico que parece ser um bom indicador da atividade inflamatória do LES. Embora não tenham observado flares durante o período do estudo, constataram um aumento da neopterina urinária sempre um dia após estressores cotidianos moderadamente intensos. Embora este não seja um achado suficiente para conclusões definitivas, os autores sugerem que possa ser um indício de que estressores psicossociais estejam realmente relacionados com a atividade inflamatória do $\operatorname{LES}^{(39)}$.

Além deste relato, merecem atenção quatro estudos de desenho transversal que observaram uma associação entre estresse e piora do LES, embora nenhum destes a tivesse como objetivo principal ${ }^{(40-43)}$.

Dois desses estudos visavam avaliar transtornos psiquiátricos em pacientes com LES, bem como a sua relação com diversas variáveis clínicas e de estresse psicossocial. O estudo de Lim et al. avaliou 40 pacientes com LES comparados a 27 pacientes com artrite reumatóide ou doença intestinal inflamatória quanto à presença de transtornos psiquiátricos, medidas de estresse social e um escore de atividade clínica e laboratorial da doença, construído pela própria equipe. As medidas de estresse social foram significativamente associadas com altos escores nas escalas de psicopatologia ou de depressão, mas não houve associação entre o estresse social e os escores de atividade clínica e laboratorial ${ }^{(40)}$. Posteriormente, Hugo et al. estudaram a prevalência de transtornos psiquiátricos e suas prováveis associações com atividade inflamatória da doença (medida através do índice do LACC), uso de corticosteróides, imagem por ressonância magnética nuclear (RMN), anormalidades no eletroencefalograma (EEG), ou eventos de vida estressantes em 88 pacientes com LES. Embora eventos da vida tenham se relacionado com a presença de transtornos psiquiátricos, não houve associação significativa entre os mesmos e variáveis orgânicas como EEG, RMN e escores do LACC ${ }^{(41)}$.

Por sua vez, os outros dois trabalhos estudaram a relação entre qualidade de vida no LES e suas variáveis clínicas e psicossociais. No primeiro destes, Dobkin et al. avaliaram 44 pacientes quanto às correlações de psicopatologia com estressores cotidianos, suporte social, qualidade de vida, atividade da doença e dano acumulado da mesma. Embora estressores tenham se associado significativamente com atividade da doença na análise univariada, esta relação não foi confirmada na análise multivariada. Interessante notar que neste estudo os estressores cotidianos correlacionaramse diretamente com sofrimento psicológico ${ }^{(42)}$. Posteriormente, este mesmo grupo avaliou a relação entre psicopatologia, estressores cotidianos e qualidade de vida e o estado de atividade do LES em 129 pacientes, que foram divididas em dois grupos de acordo com a maior e menor atividade da doença. Os autores observaram que as medidas de estresse não se relacionaram com os índices de atividade da doença avaliados pelo médico, mas sim com as medidas de saúde física avaliadas pelo paciente ${ }^{(43)}$.

É importante observar que em quase todos os estudos a presença de estresse associou-se com medidas subjetivas de sofrimento fisico ou psicológico. Porém, o tipo de desenho, o tamanho relativamente pequeno das amostras e a ausência de grupo controle são limitações evidentes destes trabalhos.

Existem seis estudos prospectivos sobre esse tema, mais adequados na verificação da correlação estudada, mas que diferem bastante quanto à metodologia empregada ${ }^{(44-49)}$. Wekking et al. seguiram 13 pacientes com LES durante um ano e mostraram que a freqüência e a intensidade de estressores diários correlacionam-se com depressão e ansiedade e número de atividades sociais, mas não com alguns parâmetros de atividade da doença (alterações laboratoriais, como hemograma e anticorpos, escalas de sintomas ou de interferência em atividades cotidianas). Apesar de serem comparados a um grupo de pacientes com artrite reumatóide como controle, o índice de drop-out deste estudo foi muito grande pois apenas cinco pacientes completaram todos os exames laboratoriais ${ }^{(44)}$. Um outro estudo, realizado através da aplicação de questionários de preenchimento diário por quase dois meses e remetidos via postal, verificou que o estresse estava associado aos sintomas de depressão, raiva e ansiedade e ao aumento da sintomatologia da doença em 41 pacientes, embora não tenha usado medidas confiáveis de piora da doença ou de presença de estressores ${ }^{(45)}$. Ambos os estudos apresentam em comum a relação do estresse com medidas subjetivas de sofrimento do paciente. 
Por outro lado, outros dois estudos avaliaram o impacto de um grande estressor ambiental nos pacientes com LES. O trabalho de Wallace et al. avaliou 10 pacientes com LES e 13 pacientes com artrite reumatóide após o terremoto que atingiu a cidade de Los Angeles em 1994. Nenhum dos pacientes apresentou exacerbações do LES após 45 dias e não foram identificadas mudanças significativas quanto à velocidade de hemossedimentação, anti-DNA, creatinina sérica e proteinúria de 24 horas ${ }^{(46)}$. Chou e Hwang também avaliaram 60 pacientes seis meses após a ocorrência de um terremoto na região onde moravam, e as compararam com 30 pacientes de outra região. Os dois grupos foram semelhantes quanto à sintomatologia clínica ou alterações laboratoriais que justificassem o diagnóstico de exacerbação. Entretanto, o grupo atingido pelo terremoto apresentou significativo aumento de creatinina e diminuição da fração C4 do complemento, que podem sugerir que o LES piorou em decorrência do impacto ambiental sofrido por estes pacientes $^{(47)}$.

Por outro lado, Dobkin et al. acompanharam 120 pacientes durante 15 meses, seguindo estressores cotidianos, sintomas psiquiátricos, qualidade de vida, suporte social e coping trimestralmente, além de atividade da doença no início e no final do estudo. Os autores verificaram que estresse não predizia aumento da atividade da doença no final. Porém, todas as pacientes eram participantes de um ensaio de psicoterapia de grupo no LES, e é provável que essa intervenção tenha interferido no impacto do estresse psicossocial sobre a doença, uma vez que todas as medidas avaliadas mostraram melhora ao longo do estudo ${ }^{(48)}$. Por fim, recentemente Pawlak et al. seguiram 41 pacientes durante 6 meses, avaliando mensalmente a ocorrência de exacerbações da doença com escores de atividade (através

\section{REFERÊNCIAS}

1. Marucha PT, Kiecolt-Glaser JK, Favagehi M: Mucosal wound healing is impaired by examination stress. Psychosom Med 60:362-5, 1998.

2. Jiang W, Babyak M, Krantz DS, et al: Mental stress-induced myocardial ischemia and cardiac events. Jama 275:1651-6, 1996.

3. Krantz DS, Santiago HT, Kop WJ, Bairey-Merz CN, Rozanski A, Gottdiener JS: Prognostic value of mental stress testing in coronary artery disease. Am J Cardiol 84:1292-7, 1999.

4. Price MA, Tennant CC, Butow PN, et al: The role of psychosocial factors in the development of breast carcinoma: Part II. Life event stressors, social support, defense style, and emotional control and their interactions. Cancer 91:686-97, 2001.

5. Lillberg K, Verkasalo PK, Kaprio J, Teppo L, Helenius H, Koskenvuo M: Stressful life events and risk of breast cancer in 10.808 women: a cohort study. Am J Epidemiol 157:415-23, 2003. do ECLAM), aumento da dose de corticóide e diminuição do complemento sérico, e da medição de estressores cotidianos. Verificaram que as exacerbações foram precedidas por aumento nos estressores em razão de relacionamentos interpessoais ocorridos no mês anterior. Além disso, também houve associação entre exacerbações do LES com aumento de estresse por obrigações sociais ${ }^{(49)}$.

\section{CONSIDERAÇÕES FINAIS}

Estudos laboratoriais sobre a presença e influência do estresse psicológico no LES mostram que há um padrão diferente de resposta imune nos pacientes, quando comparados a pessoas saudáveis, que podem estar ligados à piora ou exacerbação da doença. Além disso, estudos ambientais mostram que estressores cotidianos ligados a relacionamentos interpessoais podem preceder a piora da atividade clínica do LES. Eventos de vida maiores e estressores crônicos ainda carecem de verificação.

A pesquisa sobre a influência de estressores psicossociais no LES é recente e pouco explorada, como se vê a partir dos artigos pesquisados. As evidências são conflitantes, o que pode ser explicado pela dificuldade inerente em estudar aspectos psicológicos, ambientais e imunológicos em duas condições complexas como o LES e o estresse. Embora muitos investigadores defendam que o estresse psicológico possa deprimir a resposta imune e, conseqüentemente, agravar o estado de saúde da pessoa, a quantidade, o tipo e a duração do tempo necessários para uma mudança na resposta imune e na expressão clínica dessa mudança é ainda incerta.

A pesquisa nessa área ainda é incipiente, e mais estudos sobre o tema são necessários para responder definitivamente a essas questões.

6. Cohen S, Tyrrell DA, Smith AP: Psychological stress and susceptibility to the common cold. N Engl J Med 325:606-12, 1991.

7. Cohen S, Doyle XJ, Skoner DP: Psychological stress, cytocike production and severity of upper respiratory illness. Psychosom Med 61:175-80, 1999.

8. Leserman J, Jackson ED, Petitto JM, et al: Progression to AIDS: The effects of stress, depressive symptons, and social support. Psychosom Med 61:397-406, 1999.

9. Leserman J, Petitto JM, Gu H, et al: Progression to AIDS, a clinical AIDS condition and mortality: psychosocial and physiological predictors. Psychol Med 32:1059-73, 2002.

10. Smyth JM, Stone AA, Hurewitz A, Kaell A: Effects of writing about stressful experiences on symptom reduction in patients with asthma or rheumatoid arthritis: a randomized trial. Jama 281:1304-9, 1999.

11. Zautra AJ, Hoffman JM, Matt KS, et al: An examination of individual differences in the relationship between interpersonal stress and disease 
activity among women with rheumatoid arthritis. Arth Care Res 11:271-9, 1998.

12. Zautra AJ, Hoffman J, Potter P, Matt KS, Yocum D, Castro WL: Examination of changes in interpersonal stress as a factor in disease exacerbations among women with rheumatoid arthritis. Ann Behav Med 19:279-86, 1997.

13. Kiecolt-Glaser JK, McGuire L, Robles TF, Glaser R: Psychoneuroimmunology and Psychosomatic Medicine: Back to the Future. Psychosom Med 64:15-28, 2002.

14. Cannon WB: Bodily changes in pain, hunger, fear and rage. New York: Brandford, 1929.

15. Selye HA: A syndrome produced by diverse nocuous agents. Nature 138:517, 1936.

16. Solomon GF, Moos RH: Emotions, immunity, and disease: A speculative theoretical integration. Arch Gen Psychiatry 11:657-74, 1964.

17. Ader R, Cohen N: Behaviorally conditioned immunosupression and murine systemic lupus erythematosus. Science 214:1534-6, 1983.

18. Mello AAF, Mello MF, Carpenter LL, Price LH: Update on stress and depression: the role of the hypothalamic -pituitary-adrenal (HPA) axis. Rev Bras Psiquiatr 25:231-38, 2003.

19. Meyer SE, Chrousos GP, Gold PW: Major depression and the stress system: a life span perspective. Dev Psychopathol 13: 565-80, 2001.

20. Gold PW, Chrousos GP: Organization of the stress system and its dysregulation in melancholic and atypical depression: high vs low CRH/NE states. Mol Psychiatry 7:254-75, 2002.

21. Dimsdale JE, Keefe FJ, Stein MB: Stress and Psychiatry. In: Kaplan \& Sadock's Comprehensive Textbook of Psychiatry. Kaplan HI, Sadock BJ, Sadock VA, 7th ed, Ed Lippincott Williams \& Wilkins, 2000, cap 25.9, p 1835-46.

22. Miller AH, Pearce BD, Pariante CM: Immune system and central nervous system interaction. In: Kaplan \& Sadock's Comprehensive Textbook of Psychiatry. Kaplan HI, Sadock BJ, Sadock VA, 7th ed, Ed Lippincott Williams \& Wilkins, 2000, cap 1.12, p 113-33.

23. Naliboff BD, Solomon GF, Gilmore SL, Fahey JL, Benton D, Pine $\mathrm{J}$ : Rapid changes in cellular immunity following a confrontational role-play stressor. Brain Behav Immunity 9:207-19, 1995.

24. Glaser R, Kiecolt-Glaser JK, Bonneau RH, Malarkey W, Kennedy $\mathrm{S}$, Hughes J: Stress-induced modulation of the immune response to recombinant hepatitis B vaccine. Psychosom Med 54:22-9, 1992.

25. Vedhara K, Cos NKM, Wilcock GK, et al: Chronic stress in elderly carers of dementia patients and antibody response to influenza vaccination. Lancet 353:627-31, 1999.

26. Kiecolt-Glaser JK, Glaser R, Cacciopo JT, et al: Marital conflict in older adults: endocrinological and immunological correlates. Psychosom Med 59:339-49, 1997.

27. Arnetz BB, Wasserman J, Petrini B, et al: Immune function in unemployed women. Psychosom Med 49:3-12, 1987.

28. Arnetz BB, Brenner SO, Levi L, et al: Neuroendocrine and immunologic effects of unemployment and job insecurity. Psychother Psychosom 55:76-80, 1991.
29. Solomon GF, Segerstrom SC, Grohr P, Kemeny M, Fahey J: Shaking up immunity: psychological and immunologic changes after a natural disaster. Psychosom Med 59:114-27, 1997.

30. Kim YK, Maes M: The role of the cytokine network in psychological stress. Acta Neuropsychiatr 15:148-55, 2003.

31. Kiecolt-Glaser JK, Glaser R: Methodological issues in behavioral immunology research with humans. Brain Behav Immun 2: 67-78, 2004.

32. Petri MA: Systemic Lupus Erythematosus: Clinical Aspects. In: Koopman WJ. Arthritis and Allied Conditions - A Textbook of Rheumatology. [on line], 14th ed, Lippincott Williams \& Wilkins, 2001, cap 73, disponível em: http://www.portaldapesquisa.com.br/ databases/sites

33. Otto R, Mackay IR: Psychosocial and emotional disturbances in systemic lupus erythematosus. Med J Aust 2:488, 1967.

34. Blumenfield M: Psychological aspects of systemic lupus erythematosus. Prim Care 5:159, 1978.

35. Hall RC, Stickney SK, Gardner ER: Psychiatric symptoms in patients with systemic lupus erythematosus. Psychosom 22:15-24, 1981.

36. Hinrichsen H, Barth J, Ruckemann M, Fersfl R, Kirch W: Influence of prolonged neuropsychological testing on immunoregulatory cells and hormonal parameters in patients with systemic lupus erythematosus. Rheumatol Int 12:47-51, 1992.

37. Pawlak CR, Jacobs R, Mikeska E, et al: Patients with systemic lupus erythematosus differ from healthy controls in their immunological response to acute psychological stress. Brain Behav Imm 13: 287-302, 1999.

38. Jacobs R, Pawlak CR, Mikeska E, et al: Systemic lupus erythematosus and rheumatoid arthritis patients differ from healthy controls in their cytokine pattern after stress exposure. Rheumatol 40:868-75, 2001.

39. Schubert C, Lampe A, Rumpold G, et al: Daily psychosocial stressors interfere with the dynamics of urine neopterin in a patient with systemic lupus erythematosus: an integrative single-case study. Psychosom Med 61:876-82, 1999.

40. Lim L, Rom MA, Ormerod IEC, et al: Psychiatric and neurologic manifestations in systemic lupus erythematosus. Q J Med 249: 27-38, 1988.

41. Hugo FJ, Halland AM, Spangenberg JJ, et al: DSM-III-R Classification of psychiatric symptoms in systemic lupus erythematosus. Psychosom 37:262-9, 1996.

42. Dobkin PL, Fortin PR, Joseph L, Esdaile JM, Danoff DS, Clarke AE: Psychosocial contributors to mental and physical health in patients with systemic lupus erythematosus. Arth C Res 11:23-31, 1998.

43. Dobkin PL, Da Costa D, Dritsa M, et al: Quality of life in systemic lupus erythematosus patients during more and less active disease states: differential contributors to mental and physical health. Arth C Res 12:401-10, 1999. 
44. Wekking EM, Vingerhoets AJJM, Van Dam AP, Nossent JC, Swaak AJJG: Daily stressors and systemic lupus erythematosus: a longitudinal analysis - first findings. Psychother Psychosom 55: 108-13, 1991.

45. Adams SG, Dammers PM, Saia TL, Brantley PJ, Gaydos GR: Stress, depression and anxiety predict average symptom severity and daily symptom fluctuation in systemic lupus erythematosus. J Behav Med 17:459-77, 1994.

46. Wallace DJ, Metzger AL: Can na earthquake cause flares of rheumatoid arthritis or lupus nephritis? Arthritis Rheum 37:1826-8, 1994.
47. Chou CT, Hwang CM: Changes in the clinical and laboratory features of lupus patients after the big earthquake in Taiwan. Lupus 11:109-13, 2002

48. Dobkin PL, Da Costa D, Fortin PR, et al: Living with lupus: a prospective pan-canadian stydy. J Rheumatol 28:2442-8, 2002.

49. Pawlak CR, Witte $\mathrm{T}$, Heiken $\mathrm{H}$, et al: Flares in patients with systemic lupus erythematosus are associated with daily psychological stress. Psychother Psychosom 72:159-65, 2003. 